\title{
Phase II, Multicenter, Single-Arm, Open-Label Study to Evaluate the Efficacy and Safety of Panobinostat in Combination with Bortezomib and Dexamethasone in Japanese Patients with Relapsed or Relapsed-and-Refractory Multiple Myeloma
}

\author{
Kenshi Suzuki ${ }^{a}$ Kazutaka Sunami $^{b}$ Morio Matsumoto ${ }^{c}$ Akio Maki $^{d}$ \\ Fumika Shimada $^{d}$ Kazuyuki Suzuki $^{d}$ Kazuyuki Shimizue \\ aDepartment of Hematology, Japanese Red Cross Medical Center, Tokyo, Japan; 'Department of Hematology, \\ National Hospital Organization Okayama Medical Center, Okayama, Japan; 'Department of Hematology, National

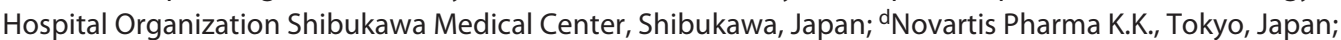 \\ eDepartment of Hematology/Oncology, Higashi Nagoya National Hospital, Nagoya, Japan
}

\section{Keywords}

Panobinostat $\cdot$ Multiple myeloma $\cdot$ Bortezomib ·

Dexamethasone

\begin{abstract}
Introduction: Panobinostat, bortezomib, and dexamethasone combination therapy demonstrated progression-free survival (PFS) benefit over bortezomib and dexamethasone alone in the PANORAMA-1 study in relapsed/refractory multiple myeloma (MM). Here, we present data from a phase II study (NCT02290431) of this combination in Japanese patients with relapsed or relapsed-and-refractory MM. Methods: Patients received 3-week cycles of 20-mg oral panobinostat (weeks 1 and 2), 1.3- $\mathrm{mg} / \mathrm{m}^{2}$ subcutaneous bortezomib (days 1, 4, 8, and 11), and 20-mg oral dexamethasone (day of and the day following bortezomib administration) for a total of 8 cycles (24 weeks; treatment phase 1). Patients with treatment benefit had an option to enter the extension phase to receive 6-week (42-day) cycles of panobinostat
\end{abstract}

karger@karger.com www.karger.com/aha

Karger ${ }^{\prime \prime} \div$

BOPEN ACCESS
(C) 2020 The Author(s)

Published by S. Karger AG, Basel

This is an Open Access article licensed under the Creative Commons Attribution-NonCommercial-4.0 International License (CC BY-NC) (http://www.karger.com/Services/OpenAccessLicense), applicable to the online version of the article only. Usage and distribution for commercial purposes requires written permission. (weeks 1, 2, 4, and 5) plus bortezomib (days 1, 8, 22, and 29) and dexamethasone (day of and the day following bortezomib treatment) for 24 weeks. The primary objective was complete response $(C R)+$ near $C R(n C R)$ rate after treatment phase 1 as per the modified European Society for Blood and Marrow Transplantation criteria. Results: Of the 31 patients, $4(12.9 \%)$ completed the treatment and $27(87.1 \%)$ discontinued; 17 (54.8\%) entered the extension phase. In total, 24 patients (77.4\%) entered the survival follow-up phase and followed until study closure when the last patient was treated for 1 year after treatment phase 1 . The $C R+n C R$ rate was 48.4\% (90\% Cl: 33.6-63.2). The overall response rate (CR + $\mathrm{nCR}+$ partial response) was $80.6 \%$. The median PFS, duration of response, time to response, and time to progression were $15.3,22.7,1.4$, and 15.3 months, respectively. All patients experienced adverse events (AEs), with diarrhea (80.6\%), decreased appetite $(58.1 \%)$, and thrombocytopenia (54.8\%) being the most frequent, regardless of relationship to the study treatment. Thrombocytopenia (48.4\%), fatigue (25.8\%), diarrhea (22.6\%), neutrophil count decrease (22.6\%), 
platelet count decrease (22.6\%), and lymphocyte count decrease (22.6\%) were the most frequent grade 3/4 AEs. Conclusion: The study met the primary objective with $48.4 \% \mathrm{CR}$ $+n C R$ rate. The AEs associated with the combination treatment were safely managed using the existing $A E$ management guidelines, including dose interruption/modification and/or supportive medical intervention. This treatment regimen is an effective option with a favorable benefit/risk profile for Japanese patients with relapsed/refractory MM.

(C) 2020 The Author(s)

Published by S. Karger AG, Basel

\section{Introduction}

Multiple myeloma is an incurable disease characterized by the proliferation of malignant plasma cells in the bone marrow along with a detection of monoclonal immunoglobulin (M-protein) in serum and/or urine $[1,2]$. Patients with multiple myeloma respond to initial therapy but succumb to the disease after multiple cycles of different kinds of therapeutic agents.

Currently, the immunomodulatory drugs (IMiDs; thalidomide, lenalidomide, and pomalidomide), proteasome inhibitors (bortezomib, carfilzomib, and ixazo$\mathrm{mib}$ ), and monoclonal antibodies (elotuzumab and daratumumab) have been employed in this disease setting [1, 3] and resulted in improvement of treatment outcome over the past decade. However, there is still a high unmet need for patients with relapsed or relapsed-and-refractory disease who have received $\geq 1$ prior therapies.

Panobinostat (LBH589), a pan-inhibitor of class I, II, and IV histone deacetylases (DACs) [4], has shown antitumor activity in preclinical models [5-8]. In the PANORAMA-1 study (CLBH589D2308) enrolling patients with relapsed or relapsed-and-refractory multiple myeloma, panobinostat in combination with bortezomib and dexamethasone showed benefit over bortezomib and dexamethasone alone in terms of progression-free survival (PFS) [9]. The safety profile was generally acceptable with dose reduction and supportive care [9]. The median PFS in the Japanese subset $(n=34)$ was longer in the panobinostat plus bortezomib and dexamethasone arm compared with the placebo plus bortezomib and dexamethasone arm (10.6 vs. 9.0 months), similar to that seen in the overall study population (12.0 vs. 8.1 months). Furthermore, although the overall response rate (ORR) was higher in the placebo arm (75.0\%) than in the panobinostat arm (61.1\%), the rate of near complete response (nCR) + complete response (CR) was higher in the panobinostat arm (33.3\%) versus the placebo arm (12.5\%) in the Japa- nese subset. In addition, the safety profile in the Japanese subset was not notably different from that in the previously reported overall population in both treatment arms and was generally manageable and predictable [10-12]. Here, we present data from a phase II study conducted to collect additional efficacy and safety data of oral panobinostat treatment in combination with bortezomib and dexamethasone in Japanese patients with relapsed or relapsed-and-refractory multiple myeloma.

\section{Methods}

\section{Patients}

This study included adult Japanese patients (age $\geq 18$ years) with relapsed multiple myeloma who received 1-5 prior lines of therapy, or with relapsed-and-refractory multiple myeloma but not refractory to prior proteasome inhibitors, requiring salvage treatment as per International Myeloma Working Group (IMWG) 2003 definitions [13]. Patients were required to have Eastern Cooperative Oncology Group (ECOG) performance status (PS) $\leq 2$; absolute neutrophil count $\geq 1.0 \times 10^{9} / \mathrm{L}$; platelet count $\geq 75 \times 10^{9} / \mathrm{L}$; serum potassium, magnesium, and phosphorus within normal limits, and total or ionized calcium (corrected for serum albumin) or ionized calcium greater than or equal to lower limit of normal, and not Common Terminology Criteria for Adverse Events (CTCAE) grade $>1$ in case of elevated value; aspartate aminotransferase and alanine aminotransferase $\leq 2.5 \times$ upper limit of normal (ULN); serum total bilirubin $\leq 1.5 \times \mathrm{ULN}$ ( or $\leq 3.0 \times \mathrm{ULN}$ if the patient has Gilbert syndrome); and estimated creatinine clearance $\geq 30 \mathrm{~mL} / \mathrm{min}$. Patients were excluded if they were receiving investigational agents, chemotherapy, biologic or immunologic therapy and/or other investigational agents, DAC inhibitors, and strong cytochrome P3A4/5 inducers.

\section{Study Design}

This was an open-label, single-arm, multicenter phase II study conducted at 21 medical centers in Japan. Patients received 20-mg panobinostat orally, thrice weekly, in combination with bortezo$\mathrm{mib}\left(1.3 \mathrm{mg} / \mathrm{m}^{2}\right.$ subcutaneously) and dexamethasone (20 mg orally). Dose adjustments (dose delay and/or reductions) were permitted at the physician's discretion based on the tolerability of the treatment.

Patients received 3-week (21-day) cycles of panobinostat (weeks 1 and 2) plus bortezomib (days 1, 4, 8, and 11) and dexamethasone (on the day of and the following day after bortezomib administration) for a total of 8 cycles ( 24 weeks; treatment phase 1). Patients who were considered to have benefited from treatment phase 1 by the investigator had an option to enter the extension treatment phase 2 to receive 6-week (42-day) cycles of panobinostat (weeks 1, 2, 4, and 5) plus bortezomib (days 1, 8, 22, and 29) and dexamethasone (on the day of and the following day after bortezomib) for 24 weeks. Clinical benefit was defined as improvement or no change at cycle 8 day 1 , as assessed by the modified European Society for Blood and Marrow Transplantation (EBMT) criteria. Patients received treatment until the end of study or until disease progression.

Dose adjustments (withholding dosing and/or reductions) were permitted, as determined by the physician according to the 
protocol-specified dose modification criteria and guidelines of panobinostat/bortezomib/dexamethasone, for patients who were unable to tolerate the protocol-specified dosing schedule. Patients who were unable to tolerate the minimum dose level of dexamethasone could continue on rest of the therapy without dexamethasone. Patients requiring the discontinuation of bortezomib due to peripheral neuropathy could continue on panobinostat and dexamethasone (or without dexamethasone). Bortezomib could be restarted at any time during the 2 phases if clinically indicated and in accordance with the local prescribing instructions for bortezomib. Panobinostat dose may be modified (20-15 and 15-10 mg/ day) during any cycle. Dose levels lower than $10 \mathrm{mg}$ thrice weekly in combination with a minimum of $0.7-\mathrm{mg} / \mathrm{m}^{2}$ bortezomib, with or without dexamethasone, are not permitted at any time of the study, and the patient will be discontinued at this stage. Patients receiving a reduced dose level of panobinostat due to toxicity may be considered for dose re-escalation according to the protocolspecified dose re-escalation criteria.

All patients were followed up throughout the treatment period and until relapse/progression after treatment discontinuation and then every 3 months for survival. Patients who discontinued study treatment due to reasons other than relapse or progression of disease were followed up for relapse/progression until 1 year after the last patient had completed 24 weeks of treatment. The study was registered on www.ClinicalTrials.gov, No. NCT02290431.

\section{Outcomes}

Primary and Secondary Endpoints

The primary endpoint was the $\mathrm{CR}+\mathrm{nCR}$ rate after all patients had been treated for 8 cycles or had discontinued treatment, based on modified EBMT criteria per investigator assessment. The secondary endpoints included PFS (time from first dose of study treatment to progression, relapse, or death due to any cause, as assessed by the investigator), ORR ( $\mathrm{CR}+\mathrm{nCR}+$ partial response [PR] based on the modified EBMT criteria per investigator assessment), overall survival (OS; time from first dose of study treatment to death), minimal response rate (MRR), time to response (TTR), time to progression/relapse (TTP), duration of response (DOR), safety, pharmacokinetics, and quality of life assessment (measured by the Functional Assessment of Cancer Therapy Gynecology Oncology Group Neurotoxicity [FACT/GOG-Ntx] scale).

Safety Assessment

Safety was routinely assessed by adverse events (AEs), serious adverse events (SAEs), physical examination, vital signs, ECOG PS, laboratory evaluations (hematology, biochemistry, coagulation, urinalysis, and thyroid function), pregnancy, electrocardiogram, and cardiac imaging. AEs were assessed according to the Common Terminology Criteria for Adverse Events (CTCAE) version 4.03 .

Pharmacokinetic Assessment

On the days of pharmacokinetic assessment (cycle 1 day 1 [C1D1] for panobinostat; cycle 1 day 8 [C1D8] for panobinostat and bortezomib), patients had a meal shortly before administration of any treatment components of panobinostat, dexamethasone, and bortezomib. Serial blood samples were collected prior to the dosing and up to $48 \mathrm{~h}$ after the dosing. The plasma concentrations of panobinostat (and the metabolite BJB432) and bortezomib were measured using validated liquid chromatography-tandem mass spectrometry. Pharmacokinetic parameters including maximum concentration $\left(C_{\max }\right)$, time to maximum concentration $\left(T_{\max }\right)$, and area under the curve (AUC) were calculated using the noncompartmental analysis method (Phoenix WinNonlin) for panobinostat, BJB432, and bortezomib.

\section{Statistical Methods}

The final analysis of the study data was performed when all patients had been treated for 8 cycles ( 24 weeks) or had discontinued prior to 8 treatment cycles. The full analysis set (FAS) included all patients who had received at least 1 dose of any study treatment component. The safety set included all patients who had received at least 1 dose of study medication (panobinostat, bortezomib, or dexamethasone). The pharmacokinetic analysis set (PAS) for panobinostat included all patients with at least 1 evaluable pharmacokinetic concentration of panobinostat.

The nCR + CR rate and its 2 -sided $90 \%$ normally approximated confidence interval (CI) were calculated. The primary analysis was based on a single-sample binomial test (normal approximation) at the 1 -sided 5\% significance level, analyzed in the FAS. The study targeted an $\mathrm{nCR}+\mathrm{CR}$ rate of $25 \%$. An $\mathrm{nCR}+\mathrm{CR}$ rate of $\leq 10 \%$ was considered as an insufficient level of activity for the study patient population. Therefore, the null $\left(H_{0}\right)$ and the alternative hypotheses $(\mathrm{Ha})$ were defined as follows: $H_{0}=\mathrm{nCR}+\mathrm{CR}$ rate $\leq 10 \%$ and $\mathrm{Ha}=\mathrm{nCR}+\mathrm{CR}$ rate $>10 \%$, respectively.

PFS was estimated using the Kaplan-Meier product-limit method and displayed as graphs. Median PFS time and its 2-sided 95\% CIs were reported.

The estimated ORR along with the corresponding 95\% Clopper-Pearson exact CIs was presented. The median TTR, DOR, and TTP along with the corresponding 95\% CIs were presented. ORR, MRR, TTR, and TTP were analyzed based on the FAS. However, DOR was analyzed based on data from responders only (CR or $\mathrm{nCR}$ or PR) in the FAS.

\section{Results}

\section{Patient Demographics and Baseline Characteristics}

The study enrolled 31 patients (median age: 68 years [range: 51-78]; female, $n=18$ ) between December 2014 and June 2017. The study was closed after the last patient had been treated for 1 year of the treatment phase 1 . The majority of the patients were aged $\geq 65$ years $(n=23$; $74.2 \%)$ and had an ECOG PS of $0(n=24 ; 77.4 \%)$. The median time since diagnosis was 47.0 months (range: $5.2-$ 133.2). According to the International Staging System (ISS), 24 (77.4\%) patients were stage I, 6 patients (19.4\%) were stage II, and 1 patient (3.2\%) was stage III. Lytic bone lesions were observed in 25 patients (80.6\%). All patients in the study had received prior anti-myeloma therapy, including 13 patients $(41.9 \%)$ who had received 1 prior line of therapy and 18 patients $(58.1 \%)$ who had received 2-3 prior lines of therapy. The most commonly used prior medications were dexamethasone $(77.4 \%)$, 
Table 1. Demographics and baseline characteristics (full analysis set)

\begin{tabular}{|c|c|}
\hline Demographic variable & $N=31$ \\
\hline Age, years, median (range) & $68(51-78)$ \\
\hline Patients aged $\geq 65$ years, $n(\%)$ & $23(74.2)$ \\
\hline Sex (female), $n(\%)$ & $18(58.1)$ \\
\hline \multicolumn{2}{|l|}{ ECOG PS, $n(\%)^{\mathrm{a}}$} \\
\hline 0 & $24(77.4)$ \\
\hline 1 & $4(12.9)$ \\
\hline 2 & $3(9.7)$ \\
\hline Time since diagnosis, months, median (range) & $47.0(5.2-133.2)$ \\
\hline Prior lines of therapy, $n$, median (range) & $2(1-3)$ \\
\hline \multicolumn{2}{|l|}{ Prior therapies (in $>50 \%$ patients), $n$ (\%) } \\
\hline Dexamethasone & $24(77.4)$ \\
\hline Bortezomib & $23(74.2)$ \\
\hline IMiDs & $20(64.5)$ \\
\hline Combined bortezomib + dexamethasone & $18(58.1)$ \\
\hline Cyclophosphamide & $17(54.8)$ \\
\hline Melphalan & $16(51.6)$ \\
\hline \multicolumn{2}{|l|}{ Involved light chain at initial diagnosis, $n(\%)$} \\
\hline Kappa & $25(80.6)$ \\
\hline Lambda & $6(19.4)$ \\
\hline \multicolumn{2}{|l|}{ Immunoglobulin isotype, $n(\%)$} \\
\hline $\operatorname{IgG}$ & $22(71.0)$ \\
\hline $\operatorname{Ig} A$ & $6(19.4)$ \\
\hline $\begin{array}{l}\text { Patients with light-chain multiple myeloma, } \\
n(\%)\end{array}$ & 4 (12.9) \\
\hline Renal function impairment, $n(\%)^{\mathrm{b}}$ & $26(83.9)$ \\
\hline Cytogenetic poor risk, $n(\%)^{\mathrm{c}}$ & $10(32.3)$ \\
\hline \multicolumn{2}{|l|}{ International Staging System, $n(\%)$} \\
\hline Stage I & $24(77.4)$ \\
\hline Stage II & $6(19.4)$ \\
\hline Stage III & $1(3.2)$ \\
\hline Patients with lytic bone lesions, $n(\%)$ & $25(80.6)$ \\
\hline
\end{tabular}

${ }^{a}$ ECOG PS: 0 = fully active, able to carry out normal activity without restriction; 1 = restricted in physical strenuous activity but ambulatory and able to carry work of a light or sedentary nature, e.g., light housework and office work; 2 = ambulatory and capable of all self-care but unable to carry out any work activities, up and about $>50 \%$ of waking hours. ECOG PS, Eastern Cooperative Oncology Group Performance Status; IMiDs, immunomodulatory drugs; CCr, creatinine clearance. ${ }^{\mathrm{b}}$ No renal function impairment: baseline $\mathrm{CCr} \geq 90 \mathrm{~mL} / \mathrm{min}$; renal function impairment: baseline $\mathrm{CCr}<90 \mathrm{~mL} / \mathrm{min}^{\mathrm{c}}$ The cytogenetic poor risk group included all patients with any of the following: cytogenetic abnormalities at baseline: $t(4,14), t(14,16), 17 p$ deletion. The cytogenetic normal risk group included all patients with none of these abnormalities at baseline.

bortezomib (74.2\%), IMiDs (64.5\%), bortezomib plus dexamethasone (58.1\%), cyclophosphamide (54.8\%), and melphalan (51.6\%). More than half of the patients (54.8\%) had received prior SCT. Table 1 describes the demographics and baseline characteristics.

Panobinostat/Bortezomib/

Dexamethasone in Patients with RRMM

\section{Patient Disposition}

Of the 31 patients treated in treatment phase 1,4 (12.9\%) completed the treatment and 27 patients $(87.1 \%)$ discontinued prematurely, primarily due to AEs $(n=20)$. In total, 17 out of the 31 patients (54.8\%) entered treatment phase 2 .

All 31 patients entered the posttreatment evaluation phase, of which 22 patients $(71.0 \%)$ discontinued from the evaluation phase, primarily due to disease progression $(n=10)$, patient/guardian decision $(n=6)$, and AEs $(n=3)$. Twenty-four patients $(77.4 \%)$ entered the survival follow-up phase (Fig. 1).

All 31 patients (100\%) were included in the FAS, safety set, and per-protocol set. The PAS of panobinostat consisted of 8 patients (25.8\%), and the PAS of bortezomib included 6 patients (19.4\%). After discontinuation of the study treatment, 21 of the 27 patients $(67.7 \%)$ received at least one antineoplastic therapy, including dexamethasone $(n=20)$, lenalidomide $(n=17)$, bortezomib $(n=8)$, daratumumab $(n=6)$, and carfilzomib $(n=6)$.

\section{Exposure}

The median duration of exposure to on-study treatment was 201 days (range: 12-985 days), with 5 patients having an exposure of $>392$ days. The median duration of exposures to panobinostat (201 days), bortezomib (197 days), and dexamethasone (198 days) were similar to the overall median duration of panobinostat plus bortezomib and dexamethasone (201 days). The majority of patients had a relative dose intensity of $<90 \%$ for panobinostat.

\section{Efficacy}

The nCR + CR rate was 48.4\% (90\% CI: 33.6-63.2), which was statistically significantly higher by $10 \%$ than that reported in the prior study $(1$-sided $p<0.0001)$. The ORR was $80.6 \%$ (95\% CI: $62.5-92.5$ ), with $25.8 \%$ of the patients achieving CR, 22.6\% achieving nCR, and 32.3\% achieving PR. The MRR was $9.7 \%$ (Table 2). The median PFS was 15.3 months (95\% CI: 10.4-31.4). Of the 15 patients who were censored, 4 were ongoing without any event, 2 withdrew consent, 8 had initiated new anticancer therapy, and 1 had an event documented after $\geq 2$ missing response assessments (Table 2; Fig. 2). The median OS was not estimable. At the time of analysis, 25 patients were alive without any event and were censored (Table 2; Fig. 2).

The median TTR was 1.4 months (95\% CI: 0.7-2.1), and the median TTP was 15.3 months (95\% CI: 10.431.4), respectively, which was consistent with the PFS findings. The median DOR was 22.7 months (95\% CI: 


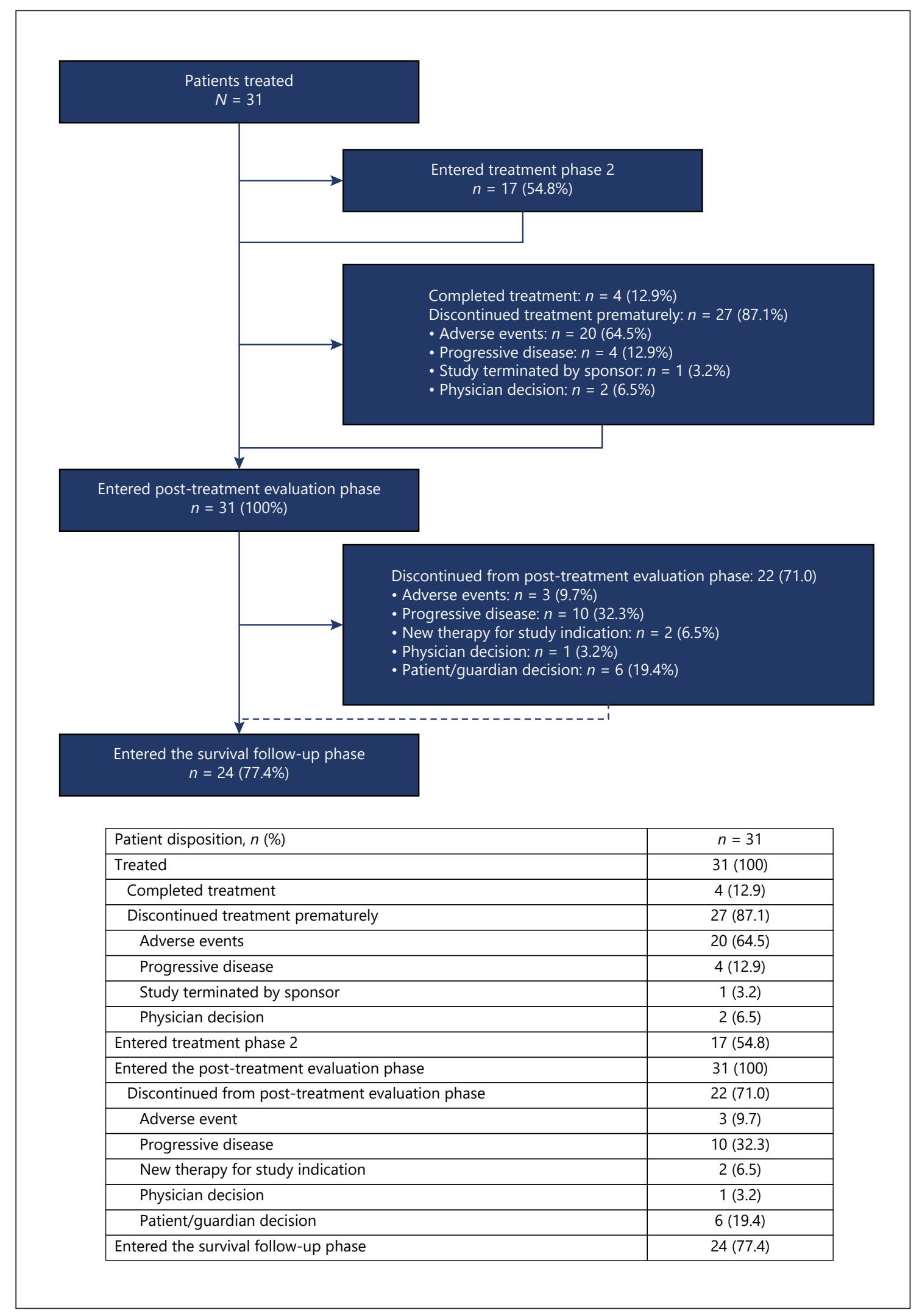

Fig. 1. Patient disposition.

Suzuki/Sunami/Matsumoto/Maki/ Shimada/Suzuki/Shimizu 


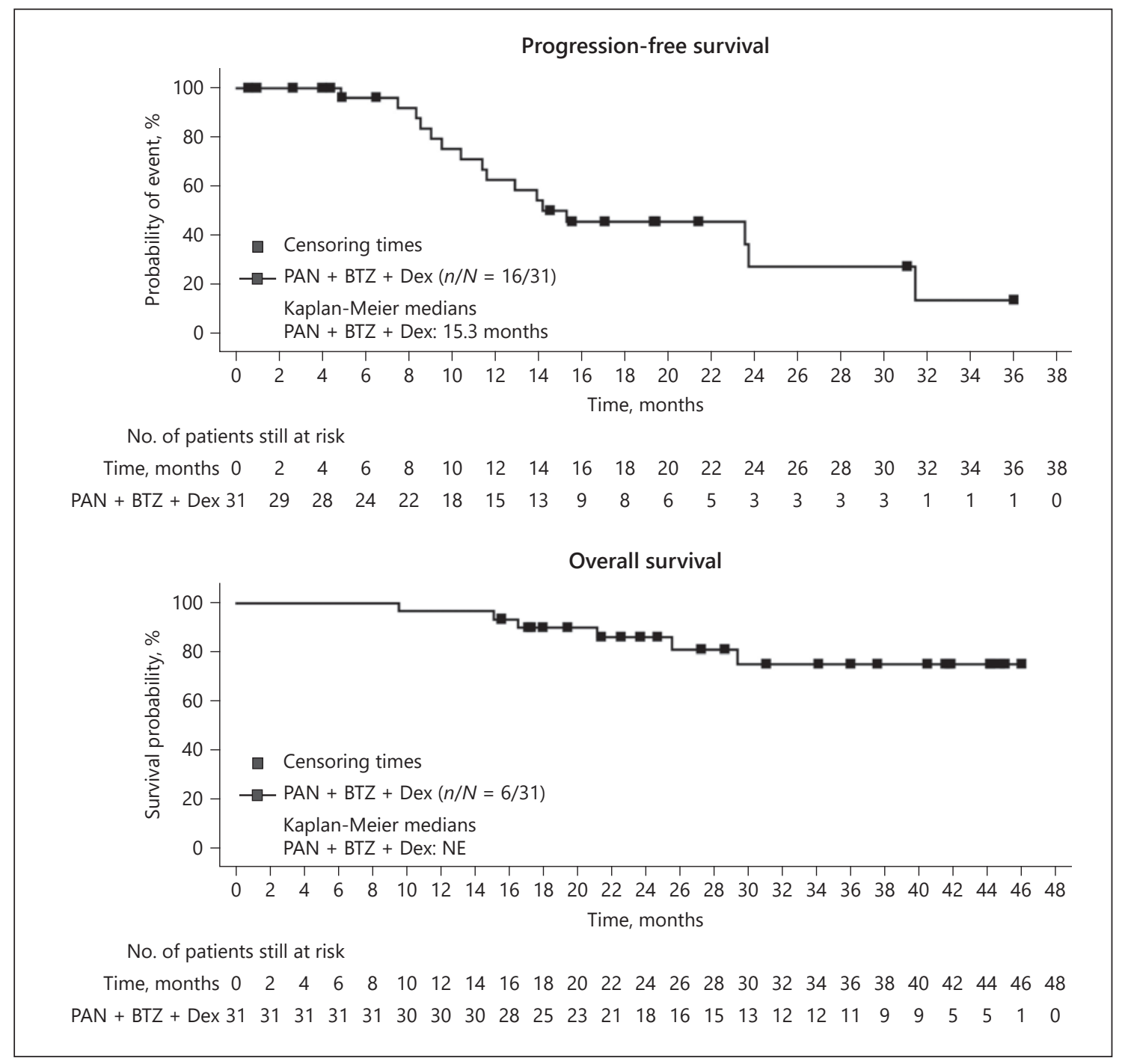

Fig. 2. Kaplan-Meier plot of progression-free survival and overall survival (full analysis set). BTZ, bortezomib; DEX, dexamethasone; PAN, panobinostat; NE, not estimable.

9.7-30.5). No on-treatment deaths were reported in the study (Table 2).

At baseline, the ECOG PS score was 0 in 24 patients (77.4\%), 1 in 4 patients (12.9\%), and 2 in 3 patients (9.7\%), respectively. During the study, ECOG PS worsened from a baseline score of 0 to $\geq 2$ in 3 patients. The FACT/GOGNtx total scores (median [range]) declined initially (112.33 [80.7-144.5] at baseline $[n=31]$ and 99.11 [65.7$147.0]$ at week $12[n=22])$, but have recovered over time during the treatment period (100.33 [59.2-149.0] at week $24[n=17], 125.83$ [91.0-149.0] at week $36[n=7]$, and 114.25 [101.7-145.0] at week 48 [ $n=6]$ ), with slight differences in the median scores at the later stages. A similar pattern was observed in the FACT/GOG-Ntx neurotoxicity subscale score. However, these results should be interpreted with caution as the number of patients decreased substantially over time.

\section{Pharmacokinetics}

The plasma concentrations of panobinostat reached its peak at $2 \mathrm{~h}$ (median $T_{\max }$ ) on both C1D1 and C1D8. On C1D1 and C1D8, the geometric mean (coefficient of variation $[\mathrm{CV}] \%)$ of $C_{\max }$ was $11.5(30.4 \%)$ and 18.2 (41.5\%) ng/mL, respectively; $\mathrm{AUC}_{0-48 \text { h was }} 106$ (14.8\%) and $156(31.7 \%) \mathrm{h} \cdot \mathrm{ng} / \mathrm{mL}$, respectively; and $T_{1 / 2}$ was 13.7 $(21.7 \%)$ and $16.5(5.2 \%)$ h, respectively. Panobinostat ex- 
posure on C1D8 was about 1.5-fold when compared with C1D1, based on $\mathrm{AUC}_{0-48 \mathrm{~h}}$ (Table 3; Fig. 3). The plasma concentrations of the metabolite BJB432 gradually increased and reached its peak at $24 \mathrm{~h}$ (median $T_{\max }$ ) on C1D1 and C1D8. From 24 to $48 \mathrm{~h}$, the concentration-time profiles were almost flat on both days. The $\mathrm{AUC}_{0-48 \mathrm{~h}}$ on C1D8 showed a 4.6-fold accumulation versus C1D1. Bortezomib plasma concentrations reached its peak rapidly, with a median $T_{\max }$ of $0.25 \mathrm{~h}$ on C1D8. The geometric mean (CV\%) of $C_{\max }$ was $43.7(22.7 \%) \mathrm{ng} / \mathrm{mL}$ and $\mathrm{AUC}_{0-48 \mathrm{~h}}$ was $188(21.1 \%) \mathrm{h} \cdot \mathrm{ng} / \mathrm{mL}$. The slope of the terminal elimination phase was slow, and therefore, the elimination half-life was not estimated within the sampling period of $48 \mathrm{~h}$.

\section{Safety}

All patients experienced at least $1 \mathrm{AE}$ during the study, and $93.5 \%$ of the patients experienced grade $3 / 4$ events. The most frequent AEs (>40\%), regardless of relationship to the study treatment, were diarrhea $(80.6 \%)$, decreased appetite (58.1\%), thrombocytopenia (54.8\%), nausea $(48.4 \%)$, and constipation (41.9\%). Thrombocytopenia (48.4\%), fatigue (25.8\%), diarrhea (22.6\%), neutrophil count decrease (22.6\%), platelet count decrease (22.6\%), and lymphocyte count decrease (22.6\%) were the most commonly reported grade $3 / 4$ events (Table 4 ). Diarrhea was managed in the majority of patients $(n=22)$ using loperamide.

No on-treatment deaths were reported in the study. The 6 posttreatment deaths were primarily due to multiple myeloma. Fourteen patients (45.2\%) experienced at least $1 \mathrm{SAE}$, with the most common SAEs being pneumonia (12.9\%), thrombocytopenia (6.5\%), and fatigue (6.5\%). No significant incidence of thyroid dysfunction was reported, and no patient had a QTcF interval of $>480$ ms. QTcF prolongation events ( $>450$ and $\leq 480 \mathrm{~ms}$ ) were observed in 2 patients (6.5\%), and 1 patient (3.2\%) experienced grade $3 / 4$ event of syncope, which required dose adjustment or temporary interruption. None of the pa-
Table 2. Efficacy result (full analysis set)

\begin{tabular}{|c|c|}
\hline Parameter & $\begin{array}{l}\text { Relapsed/ } \\
\text { refractory } \\
\text { multiple } \\
\text { myeloma, } N=31\end{array}$ \\
\hline \multicolumn{2}{|l|}{ Best overall response, $n(\%)^{\mathrm{a}}$} \\
\hline $\mathrm{CR}$ & $8(25.8)$ \\
\hline $\mathrm{nCR}$ & $7(22.6)$ \\
\hline PR & $10(32.3)$ \\
\hline Minimal response & $3(9.7)$ \\
\hline No change & $3(9.7)$ \\
\hline $\mathrm{CR} / \mathrm{nCR}, n(\%) ; 90 \% \mathrm{CI}^{\mathrm{b}}$ & $15(48.4)$ \\
\hline Overall response $(\mathrm{CR}+\mathrm{nCR}+\mathrm{PR}), n(\%)$; & $\begin{array}{l}33.6-63.2 \\
25(80.6) ;\end{array}$ \\
\hline $95 \%$ exact $\mathrm{CI}^{\mathrm{c}}$ & $62.5-92.5$ \\
\hline Time to response, months ( $95 \% \mathrm{CI})$ & $1.4(0.7-2.1)$ \\
\hline Duration of response, months ( $95 \% \mathrm{CI})$ & $22.7(9.7-30.5)$ \\
\hline Time to progression, months $(95 \% \mathrm{CI})$ & $15.3(10.4-31.4)$ \\
\hline \multicolumn{2}{|l|}{ Progression-free survival } \\
\hline Events, $n(\%)$ & $16(51.6)$ \\
\hline Patients censored, $n(\%)$ & $15(48.4)$ \\
\hline \multicolumn{2}{|l|}{ Kaplan-Meier estimate, months $(95 \% \mathrm{CI})^{\mathrm{d}}$} \\
\hline 25th percentile probability & $10.4(7.5-13.9)$ \\
\hline Median & $15.3(10.4-31.4)$ \\
\hline 75th percentile probability & $31.4(23.6-\mathrm{NE})$ \\
\hline \multicolumn{2}{|l|}{ Overall survival } \\
\hline Events, $n(\%)$ & $6(19.4)$ \\
\hline Patients censored, $n(\%)$ & $25(80.6)$ \\
\hline \multicolumn{2}{|l|}{ Kaplan-Meier estimate, months $(95 \% \mathrm{CI})^{\mathrm{d}}$} \\
\hline 25 th percentile probability & $\mathrm{NE}(16.5-\mathrm{NE})$ \\
\hline Median & $\mathrm{NE}(\mathrm{NE}-\mathrm{NE})$ \\
\hline 75th percentile probability & $\mathrm{NE}(\mathrm{NE}-\mathrm{NE})$ \\
\hline
\end{tabular}

$N$, the total number of patients in the treatment group. It was the denominator for percentage (\%) calculation. $n$, number of patients who were at the corresponding category. $\mathrm{CR}$, complete response; $\mathrm{nCR}$, near CR; PR, partial response; CI, confidence interval; EBMT, European Society for Bone and Marrow Transplantation; NE, not estimable. ${ }^{a}$ Responses were assessed as per modified EBMT criteria. ${ }^{\mathrm{b}}$ The CI was computed using the normal approximation method. ${ }^{c}$ The $95 \%$ exact CIs, for the frequency distribution of each variable, were computed using the Clopper-Pearson method. ${ }^{\mathrm{d}}$ Percentiles with 95\% CIs were calculated from the PROC LIFETEST output using the method of Brookmeyer and Crowley (1982).

Table 3. Summary of plasma panobinostat pharmacokinetic parameters by day (pharmacokinetic analysis set)

\begin{tabular}{lcc}
\hline Pharmacokinetic parameter & Cycle 1 day $1, n=8$ & Cycle 1 day $8, n=7$ \\
\hline $\mathrm{AUC}_{0-48 \mathrm{~h}}, \mathrm{~h} \cdot \mathrm{ng} / \mathrm{mL}$, geometric mean $(\mathrm{CV} \%)$ & $106(14.8)$ & $156(31.7)$ \\
$C_{\max }, \mathrm{ng} / \mathrm{mL}$, geometric mean $(\mathrm{CV} \%)$ & $11.5(30.4)$ & $18.2(41.5)$ \\
$T_{\max }, \mathrm{h}$, median (range) & $2(1.00-4.00)$ & $2(0.533-3.08)$ \\
$T_{1 / 2}, \mathrm{~h}$, geometric mean $(\mathrm{CV} \%)$ & $13.7(21.7)$ & $16.5(5.2)$ \\
\hline
\end{tabular}

AUC, area under the curve; $C_{\max }$, maximum concentration; $T_{\max }$, time to maximum concentration; CV\%, coefficient of variation. 


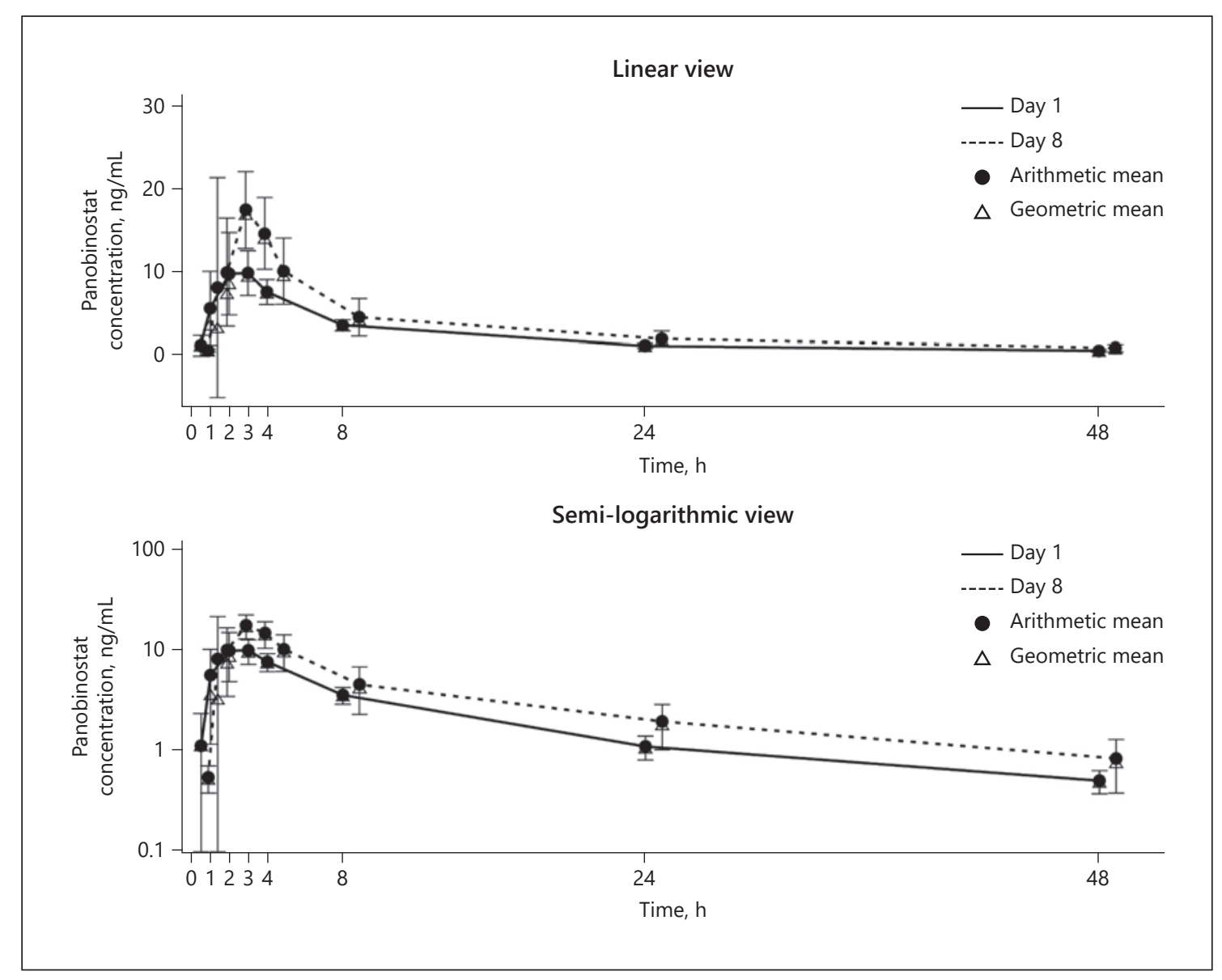

Fig. 3. Plasma panobinostat concentration-time profiles in combination with bortezomib and dexamethasone at cycle 1 day 1 and cycle 1 day 8 (panobinostat pharmacokinetic analysis set): arithmetic mean \pm SD and geometric mean (zero concentrations at individual timepoints were excluded from geometric mean computation).

tients experienced an SAE or discontinued the study due to QTc prolongation.

A total of 20 patients (64.5\%) discontinued treatment due to AEs, including fatigue (16.1\%), diarrhea (9.7\%), decreased appetite $(9.7 \%)$, nausea $(6.5 \%)$, and pneumonia (6.5\%). AEs requiring dose adjustment or study drug interruption were reported in 30 patients $(96.8 \%)$, with thrombocytopenia (35.5\%), diarrhea (19.4\%), malaise (19.4\%), decreased appetite (19.4\%), and neutropenia (16.1\%) being the most common reasons.

\section{Discussion/Conclusion}

In this phase II study, the efficacy and safety of oral panobinostat treatment in combination with bortezomib and dexamethasone in Japanese patients with relapsed/ refractory multiple myeloma were studied. Key patient selection criteria were similar to the pivotal global phase III study (PANORAMA-1) that demonstrated a survival benefit of panobinostat over placebo in combination with bortezomib and dexamethasone [9].

This study met its primary objective demonstrating an $\mathrm{nCR}+\mathrm{CR}$ rate of $>10 \%$, with an observed $\mathrm{nCR}+\mathrm{CR}$ rate of $48.4 \%$ (90\% CI: 33.6-63.2) per investigator assessment. The median PFS of 15.3 months and ORR of $80.6 \%$ observed in this study provide supportive evidence that the benefit for Japanese patients is comparable to the overall population in the PANORAMA-1 study (PFS: 12.0 months; ORR: 60.7\%) [9].

No on-treatment deaths were reported in the study. There were 6 OS events reported, and 25 patients were censored as they were alive without any event. At the time of the final assessment, the median OS was not estimable. 
Table 4. Adverse events (all grades occurring in $\geq 10 \%$ of patients), regardless of the study treatment relationship by preferred term (safety set)

\begin{tabular}{lll}
\hline Preferred term ${ }^{\text {a,b }}$ & \multicolumn{2}{l}{ Relapsed/refractory multiple } \\
& myeloma, $N=31$ & \\
\cline { 2 - 3 } & all grades, & grade 3/4, \\
& $n(\%)$ & $n(\%)$ \\
\hline Diarrheac, & $25(80.6)$ & $7(22.6)$ \\
Decreased appetite & $18(58.1)$ & $5(16.1)$ \\
Thrombocytopenia & $17(54.8)$ & $15(48.4)$ \\
Nausea & $15(48.4)$ & $2(6.5)$ \\
Constipation & $13(41.9)$ & $1(3.2)$ \\
Malaise & $11(35.5)$ & $1(3.2)$ \\
Anemia & $10(32.3)$ & $6(19.4)$ \\
Fatigue & $10(32.3)$ & $8(25.8)$ \\
Neutrophil count decreased & $9(29.0)$ & $7(22.6)$ \\
Platelet count decreased & $9(29.0)$ & $7(22.6)$ \\
Vomiting & $9(29.0)$ & 0 \\
Peripheral edema & $8(25.8)$ & $1(3.2)$ \\
Pyrexia & $8(25.8)$ & $1(3.2)$ \\
Dysgeusia & $7(22.6)$ & 0 \\
Insomnia & $7(22.6)$ & 0 \\
Lymphocyte count decreased & $7(22.6)$ & $7(22.6)$ \\
Nasopharyngitis & $7(22.6)$ & 0 \\
Neutropenia & $7(22.6)$ & $6(19.4)$ \\
Hypokalemia & $6(19.4)$ & $6(19.4)$ \\
Peripheral neuropathy & $6(19.4)$ & $1(3.2)$ \\
Herpes zoster reactivation & $5(16.1)$ & $1(3.2)$ \\
Hypertension & $5(16.1)$ & $2(6.5)$ \\
Hypophosphatemia & $5(16.1)$ & $5(16.1)$ \\
Lymphopenia & $5(16.1)$ & $3(9.7)$ \\
Peripheral sensory neuropathy & $5(16.1)$ & $1(3.2)$ \\
Pneumonia & $5(16.1)$ & $3(9.7)$ \\
Rash & $5(16.1)$ & $1(3.2)$ \\
Upper respiratory tract infection & $5(16.1)$ & 0 \\
Arthralgia & $4(12.9)$ & 0 \\
Hepatic function abnormal & $4(12.9)$ & $2(6.5)$ \\
Leukopenia & $4(12.9)$ & $3(9.7)$ \\
Weight loss & $4(12.9)$ & 0 \\
White blood cell count decreased & $4(12.9)$ & $3(9.7)$ \\
\hline & & \\
\hline & & \\
& &
\end{tabular}

${ }^{a}$ A patient with multiple occurrences of an adverse event under one treatment was counted only once in the adverse event category for that treatment. ${ }^{\mathrm{b}}$ According to MedDRA, version 21.1, Common Terminology Criteria for Adverse Events version 4.03. ${ }^{\mathrm{c}}$ Diarrhea was managed in the majority of patients $(n=22)$ using loperamide. Loperamide $4 \mathrm{mg}$ was to be taken at the onset of diarrhea, followed by $2-\mathrm{mg}$ dose as needed, no more frequently than every $4 \mathrm{~h}$, not exceeding a total of $16 \mathrm{mg}$ in $24 \mathrm{~h}$. Patients with grade $\geq 2$ diarrhea despite loperamide treatment were to interrupt panobinostat treatment. If the above intervention is inadequate, additional evaluation and treatments were to be pursued as medically indicated. Replacement intravenous fluids and electrolytes may be used as appropriate. Additional treatment was to be provided in accordance with institutional standard of care and/or local guidelines. ${ }^{\mathrm{d}}$ Diarrhea incidences in the majority of the patients ( 24 of 25 patients) were suspected to be related to study treatment. Seven patients (22.6\%) experienced grade $3 / 4$ events, and no patient experienced serious events. Six patients (19.4\%) had dose adjustment or temporary interruption of study treatment, and 3 patients (9.7\%) discontinued the study due to diarrhea.
Japanese patients in this study, versus patients in the PANORAMA-1 study, had comparable median TTR (1.4 vs. 1.5 months), median TTP (15.3 vs. 12.7 months), and relatively longer median DOR (22.7 vs. 13.1 month) [9]. The pharmacokinetic profile of panobinostat in this study was similar to that observed in the PANORAMA-1 study. Also for bortezomib, although the route of administration was different in this study (subcutaneous) compared with the PANORAMA-1 study (intravenous), the drug exposure $\left(\mathrm{AUC}_{0-48 \mathrm{~h}}\right)$ was similar [9].

The safety and tolerability profile of panobinostat plus bortezomib and dexamethasone observed in the current study was generally consistent with prior experience; panobinostat in combination with bortezomib and dexamethasone was associated with a manageable and predictable safety profile. The overall results indicate that the majority of AEs can be safely managed with the use of existing AE management guidelines. The toxicities observed with panobinostat in combination with bortezomib and dexamethasone were likely due to the known overlapping toxicities, particularly myelosuppression, diarrhea, and fatigue. These events, however, were successfully managed with dose interruption, dose modification, and/or supportive medical intervention.

In addition, infection is a known risk for patients with multiple myeloma as a result of disease-associated immunosuppression [14-16]. The incidence of AEs leading to discontinuation of study treatment was $64.5 \%$. AEs requiring dose adjustment or study drug interruption were reported in a higher proportion of patients $(96.8 \%)$. The relatively higher frequencies of AEs requiring dose adjustment or interruption, coupled with much lower frequencies of such AEs leading to study discontinuation, suggest that dose adjustment or interruption is one of the effective approaches to manage AEs with this treatment regimen. In general, treatment with panobinostat plus bortezomib and dexamethasone was associated with an increased risk of thrombocytopenia, infection (mainly pneumonia), diarrhea, and fatigue in patients with multiple myeloma. Most of the patients reported thrombocytopenia and diarrhea events. However, QTc prolongation and pneumonia incidences were minimal and there were no incidents of hemorrhage. No additional or new safety concerns were identified in Japanese patients in this study.

In the context of the significant clinical benefit observed in this patient population, who often are without effective treatment options and with clinically manageable thrombocytopenia, diarrhea, and fatigue, this treatment regimen is considered to be a valuable option with a favorable benefit/risk profile for Japanese patients with 
relapsed or relapsed-and-refractory multiple myeloma who have received at least 1 prior therapy. Panobinostat is being investigated in other novel combinations with either carfilzomib ixazomib, or lenalidomide in this patient population who has limited options $[17,18]$.

\section{Acknowledgements}

We thank the patients and their families, the study investigators, and the study site personnel for their participation and contributions to this study. We would thank Vijay Kadasi, MSc, Novartis Healthcare Pvt Ltd, for providing medical writing and editorial assistance support.

\section{Statement of Ethics}

This study was conducted according to the International Conference on Harmonization Harmonized Tripartite Guidelines-E6 Guideline for Good Clinical Practice that have their origin in the Declaration of Helsinki and applicable local regulations. Informed consent was obtained from all individual participants included in the study. The protocol and informed consent forms were reviewed and approved by an institutional review board, independent ethics committee, or research ethics board before the study start at each participating institution.

\section{Conflict of Interest Statement}

Kenshi Suzuki received honoraria from Celgene, Takeda, Ono pharmaceutical, Amgen, Novartis, Sanofi, Bristol-Myers Squibb, AbbVie, and Janssen; acted as a consultant for Amgen,
Janssen, Takeda, and Celgene; and received research funding from Bristol-Myers Squibb, Celgene, and Amgen. Kazutaka Sunami received research funding from Novartis, GlaxoSmithKline, Janssen, AbbVie, Takeda, Sanofi, Bristol-Myers Squibb, Ono pharmaceutical, MSD, Alexion Pharma, Daiichi Sankyo, and Celgene and received honoraria from Takeda, Bristol-Myers Squibb, Ono pharmaceutical, and Celgene. Morio Matsumoto received personal fee from Bristol-Myers Squibb K.K., Janssen Pharmaceutical Co., Ltd, Celgene Co. Ltd, and Ono Pharmaceutical Co. Ltd. Akio Maki, Fumika Shimada, and Kazuyuki Suzuki are employees of Novartis; Kazuyuki Shimizu has nothing to disclose.

\section{Funding Sources}

This study was sponsored by Novartis Pharma K.K., Japan.

\section{Author Contributions}

Akio Maki, Fumika Shimada, Kazuyuki Suzuki, and Kazuyuki Shimizu contributed to conceptualization and design of the study and data interpretation. Kenshi Suzuki, Kazutaka Sunami, and Morio Matsumoto contributed to patient accrual, clinical care, and data collection. Kazuyuki Suzuki was involved in trial management. Fumika Shimada is the trial statistician. Akio Maki, Fumika Shimada, and Kazuyuki Suzuki were involved in data analysis. Kazuyuki Shimizu was the PI of this study leading to the approval of panobinostat in Japan by addressing queries provided the PMDA and reviewed the final version of the draft. All authors contributed to drafting, revising, and final review and approval of the manuscript.

\section{References}

1 Moreau P, San Miguel J, Sonneveld P, Mateos MV, Zamagni E, Avet-Loiseau H, et al. Multiple myeloma: ESMO Clinical Practice Guidelines for diagnosis, treatment and follow-up. Ann Oncol. 2017;28(Suppl 4):iv5261.

2 Palumbo A, Anderson K. Multiple myeloma. N Engl J Med. 2011;364(11):1046-60.

3 Ozaki S, Handa H, Saitoh T, Murakami H, Itagaki M, Asaoku H, et al. Trends of survival in patients with multiple myeloma in Japan: a multicenter retrospective collaborative study of the Japanese Society of Myeloma. Blood Cancer J. 2015;5:e349.

4 Atadja P. Development of the pan-DAC inhibitor panobinostat (LBH589): successes and challenges. Cancer Lett. 2009;280(2): 233-41.

5 Maiso $\mathrm{P}$, Carvajal-Vergara X, Ocio EM, López-Pérez R, Mateo G, Gutiérrez N, et al. The histone deacetylase inhibitor LBH589 is a potent antimyeloma agent that overcomes drug resistance. Cancer Res. 2006;66(11): 5781-9.

6 Catley L, Weisberg E, Kiziltepe T, Tai YT, Hideshima T, Neri P, et al. Aggresome induction by proteasome inhibitor bortezomib and alpha-tubulin hyperacetylation by tubulin deacetylase (TDAC) inhibitor LBH589 are synergistic in myeloma cells. Blood. 2006; 108(10):3441-9.

7 Growney JD, Atadja P, Shao W, Wang Y, Pu $M$, Firestone B, et al. Efficacy of panobinostat (LBH589) in multiple myeloma cell lines and in vivo mouse model: tumor-specific cytotoxicity and protection of bone integrity in multiple myeloma. Blood. 2007;110(11): 452a.

8 Kalushkova A, Fryknäs M, Lemaire M, Fristedt C, Agarwal P, Eriksson M, et al. Polycomb target genes are silenced in multiple myeloma. PLoS One. 2010;5(7):e11483.
9 San-Miguel JF, Hungria VT, Yoon SS, Beksac M, Dimopoulos MA, Elghandour A, et al. Overall survival of patients with relapsed multiple myeloma treated with panobinostat or placebo plus bortezomib and dexamethasone (the PANORAMA 1 trial): a randomised, placebo-controlled, phase 3 trial. Lancet Haematol. 2016;3(11):e506-15.

10 San-Miguel JF, Hungria VT, Yoon SS, Beksac M, Dimopoulos MA, Elghandour A, et al. Panobinostat plus bortezomib and dexamethasone versus placebo plus bortezomib and dexamethasone in patients with relapsed or relapsed and refractory multiple myeloma: a multicentre, randomised, double-blind phase 3 trial. Lancet Oncol. 2014;15(11):1195-206.

11 Evaluation and Licensing Division, Pharmaceutical and Food Safety Bureau Ministry of Health, Labour and Welfare. Farydak Review Report. 2015. Accessed 2019 Nov 13. Available from: https://www.pmda.go.jp/files/000212785.pdf. 
12 Richardson PG, Hungria VTM, Yoon S-S, Beksac M, Dimopoulos MA, Elghandour A, et al. Panorama 1: a randomized, double-blind, phase 3 study of panobinostat or placebo plus bortezomib and dexamethasone in relapsed or relapsed and refractory multiple myeloma. J Clin Oncol. 2014;32(15 Suppl):8510.

13 International Myeloma Working Group. Criteria for the classification of monoclonal gammopathies, multiple myeloma and related disorders: a report of the International Myeloma Working Group. Br J Haematol. 2003;121(5): 749-57.
14 Blimark C, Holmberg E, Mellqvist UH, Landgren $\mathrm{O}$, Björkholm $\mathrm{M}$, Hultcrantz $\mathrm{M}$, et al. Multiple myeloma and infections: a population-based study on 9253 multiple myeloma patients. Haematologica. 2015;100(1):10713.

15 Torti L, Morelli A, Bacci F, Di Bartolomeo P. Infections and immune system impairment in multiple myeloma: increasing frequency of serious complications in the "novel agents era": a retrospective real life analysis. Blood. 2017;130(Suppl 1):1875.

16 Nucci M, Anaissie E. Infections in patients with multiple myeloma in the era of high-dose therapy and novel agents. Clin Infect Dis. 2009;49(8):1211-25.
17 Kaufman JL, Mina R, Jakubowiak AJ, Zimmerman TL, Wolf JJ, Lewis C, et al. Combining carfilzomib and panobinostat to treat relapsed/refractory multiple myeloma: results of a Multiple Myeloma Research Consortium Phase I Study. Blood Cancer J. 2019;9(1):3.

18 Reu FJ, Valent J, Malek E, Sobecks RM, Faiman BM, Hamilton K, et al. A phase I study of ixazomib in combination with panobinostat and dexamethasone in patients with relapsed or refractory multiple myeloma. Blood. 2015;126(23). 